\title{
A 7 Tesla fMRI Study of Amygdala Responses to Fearful Faces
}

\author{
Wietske van der Zwaag $\cdot$ Sandra E. Da Costa $\cdot$ \\ Nicole R. Zürcher • Reginald B. Adams Jr. • \\ Nouchine Hadjikhani
}

Received: 22 July 2011/ Accepted: 7 January 2012/Published online: 22 January 2012

(C) The Author(s) 2012. This article is published with open access at Springerlink.com

\begin{abstract}
The amygdalae are involved in the perception of emotions such as happiness, anger and fear. Because of their proximity to the sinuses, the image signal intensity in T2* weighted fMRI data is often affected by signal loss due to through-slice dephasing, especially at high field strength. In this study, the feasibility of fMRI in the amygdalae at 7 Tesla was investigated. A paradigm based on the presentation of fearful faces was used for stimulation. Previously, opposite effects have been found for presentation of averted and direct gaze fearful faces. Here, we show that (1) sufficiently high temporal SNR values are reached in the amygdalae for detection of small BOLD signal changes and (2) that the BOLD signal in the amygdalae for presentation of a direct or averted gaze in a fearful face depends on stimulus duration.
\end{abstract}

Keywords Amygdala fMRI $\cdot 7$ Tesla $\cdot$ Fear . Face perception

W. van der Zwaag

Department of Radiology, University of Lausanne, Lausanne, Switzerland

S. E. Da Costa · N. R. Zürcher · N. Hadjikhani

Brain Mind Institute, EPFL, Lausanne, Switzerland

R. B. Adams Jr.

Department of Psychology, The Pennsylvania State University, University Park, USA

N. Hadjikhani

MGH/HMS/HST Martinos Center for Biomedical Imaging,

Boston, USA

N. Hadjikhani ( $\square)$

GRHAD, Station 19, 1015 Lausanne, Switzerland

e-mail: nouchine@nmr.mgh.harvard.edu

\section{Introduction}

As the BOLD signal is based on the susceptibility difference between deoxyhaemoglobin and surrounding tissue, BOLD-based fMRI sees a significant signal increase with field strength (van der Zwaag et al. 2009; Yacoub et al. 2001). This signal increase is typically traded for higher spatial resolution or shorter scan times. As fMRI studies of the amygdala generally report small signal changes (Norbury et al. 2010), the use of ultra-high field could be advantageous. Unfortunately, the amygdalae are in a brain region which can be severely affected by through-slice dephasing and susceptibility induced distortions in gradient echo EPI (Merboldt et al. 2001), which also increase with field strength. Therefore, the use of relatively small voxel size to limit the susceptibility-induced distortions and a thin slice thickness in combination with careful slice positioning to avoid through-slice dephasing become even more important at higher fields. As the main magnetic field gradients are found in the cranio-caudal and anterior-posterior directions, a pseudo-coronal slice orientation is generally found to be optimal (Merboldt et al. 2001; Robinson et al. 2004).

A fearful face signals to the viewer the presence of a threat, thus inducing fear, although the exact mechanisms remain to be determined. Amygdala activation is known to be modulated by the perception of fearful faces (Ohrmann et al. 2007) and the relevance thereof for the observer (Cristinzio et al. 2010). Relevance can be changed by modifying the gaze direction of the presented face. Studies using similar stimuli of fearful faces (Adams et al. 2003; Hadjikhani et al. 2008; N'Diaye et al. 2009) have reported opposite results. While Adams et al. reported signal increases in the amygdalae for direct versus averted fearful faces (Adams et al. 2003), others reported BOLD signal 
increases for presentations of averted vs direct fearful faces (Hadjikhani et al. 2008; N'Diaye et al. 2009). However, these studies used different cohorts of subjects, different sets of stimuli and different presentation parameters, all of which can modulate the amygdale response. A recent study, using a between subject design, suggested that this apparent discrepancy is due to stimulus duration (Adams et al. 2011). Here, the same fearful faces are presented a within subjects design during two separate runs in a single scanning session at 7 Tesla, varying only the stimulus presentation time.

\section{Methods}

Eighteen healthy volunteers (10 males, mean age 24 years) passively observed fearful faces with direct or averted gaze in a short $(300,1,200 \mathrm{~ms}$ ISI) or a long $(1,500,0 \mathrm{~ms} \mathrm{ISI})$ presentation paradigm. Stimuli were identical to those previously used by Hadjikhani et al. (2008), taken from the NimStim Emotional Face Stimuli database. 24 s blocks of averted (4 blocks left/4 blocks right) and direct gaze were presented in a pseudo random order during two runs of $384 \mathrm{~s}$ each. All procedures were approved by Lausanne University ethical committee and all subjects provided written consent.

Data were acquired on a 7 Tesla scanner (Siemens, Germany) with eight channel rf-coil (Rapid Biomedical, Germany). A multi-slice EPI sequence (Speck et al. 2008) with sinusoidal read-out, to limit the helium boil-off, was used for functional acquisition (40 $1.5 \mathrm{~mm}$ thick slices, oriented $30^{\circ}$ from $\mathrm{AC}-\mathrm{PC}, \mathrm{TR}=3,000 \mathrm{~ms}, \mathrm{TE}=21 \mathrm{~ms}$, flip $60^{\circ}$, FOV $160 \mathrm{~mm}^{2}$, matrix $64 \times 64,2.5 \times 2.5 \times$ $1.5 \mathrm{~mm}$ ). Slices were positioned at an approximately $30^{\circ}$ angle from the AC-PC line (Robinson et al. 2004), while ensuring that the eyes and mobile parts of the brainstem were not included in the imaging slab to avoid motioninduced Nyquist ghosting (Fig. 1b). The relatively thin slice thickness was chosen to further reduce through-slice signal dephasing in the medial part of the amygdalae.

A single full brain EPI volume (100 slices, TR $=$ $6,000 \mathrm{~ms}$ ) was acquired to aid co-registration with the anatomical images acquired with MP2RAGE ((Marques et al. 2010), $\mathrm{TR}=6.9 \mathrm{~ms}$, TE $=2.82 \mathrm{~ms}$, $\mathrm{TR}=5,000 \mathrm{~ms}$, $\mathrm{TI}_{1} / \mathrm{TI}_{2}=700 / 2,500 \mathrm{~ms}$, flip $4^{\circ} / 5^{\circ}$, FOV $256 \mathrm{~mm}^{2}$, matrix size $256 \times 240$ ).

Images were analyzed using mixed-effect with FSL FEAT (www.fmrib.ox.ac.uk/fsl), after motion correction, spatial smoothing (FWHM $=6.0 \mathrm{~mm}$, to ensure equal smoothness after spatial normalisation), high pass temporal filtering $(\sigma=50 \mathrm{~s})$ and co-registration to the MNI brain with FLIRT.
Temporal SNR, tSNR, maps were obtained per subject by dividing the mean of each voxel timecourse by its temporal standard deviation after motion correction, smoothing and temporal filtering.

\section{Results and Discussion}

TSNR was, on average over an amygdala ROI taken from the Harvard-Oxford atlas, $116 \pm 7$ (mean \pm stderr); high enough to detect the small BOLD signal changes expected in the amygdalae. $90 \pm 2 \%$ of the amygdalae ROI fell in areas with tSNR $>50$. A tSNR map from a representative subject is shown in Fig. 1b.

Bilateral amygdala activation (maximum at: MNI 24, $-4,-20: Z=2.28$ and $-22,-7,-18: Z=2.36$ ) was found for short duration averted versus direct presentations (Fig. 2a), in agreement with the signal changes reported by Hadjikhani et al. (2008) for the left amygdale and as reported for dynamic stimuli in (N'Diaye et al. 2009). This effect was reversed, however, in the same subject cohort, for long duration direct versus averted presentations (maximum at: MNI 15, $-9,-19: \mathrm{Z}=2.76$ and $-17,-18$, $-20: Z=3.06$, Fig. 2 b), in accordance with results from Adams et al. (2003) in the left amygdala. A direct comparison revealed a significant difference for the influence of gaze in short versus long presentations, (maximum at: MNI $15,-9,-19: Z=3.15$ and $-15,-8,-19: Z=3.06)$ with greater response to averted minus direct for short presentations and to direct minus averted for long presentations (Fig. 2c). That activation is found bilaterally in this study as opposed to previous reports (Adams et al. 2011; Adams et al. 2003; Hadjikhani et al. 2008) is likely due to the higher BOLD sensitivity at 7 Tesla or the more liberal threshold. The maximum $z$-score for both short and long duration stimuli is slightly higher in the left amygdala, in agreement with previous work. Bilateral activation has previously been reported for related emotional fMRI tasks (N'Diaye et al. 2009).

An ISI was introduced in the short-presentation protocol to allow block timing and the number of presented stimuli to be the same between the two protocols, hence ISIs varied across duration conditions. However, the within-run BOLD contrasts always used the same ISI for both averted and direct gaze, and thus processing during ISI was not thought to influence the results; although further testing, with stimulus durations constant and varying ISI, would be needed to confirm this.

Areas involved in joint attention included in the images (frontal operculum, inferior parietal cortex and insula) showed higher activation for averted gaze regardless of stimulus duration. 


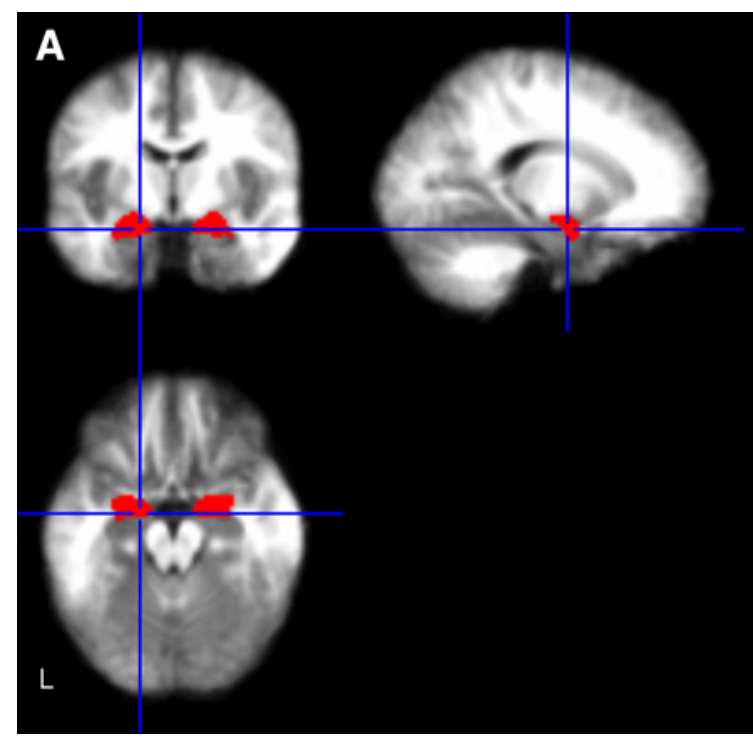

Fig. 1 a Amygdalae from the Harvard-Oxford subcortical atlas. b Temporal SNR map from a representative subject for the area of the fMRI acquisition volume. Shown tSNR values range from 50 to 300 .

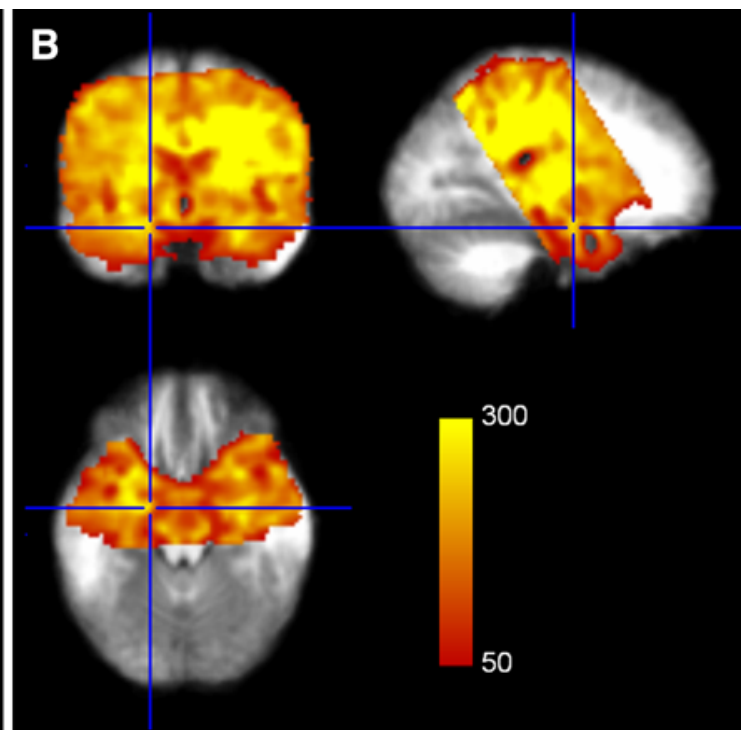

Crosshair lines are positioned at $-18,-5,-17$ in MNI space. Note that tSNR in the medial part of the amygdala is lower than in the lateral region due to through-slice dephasing
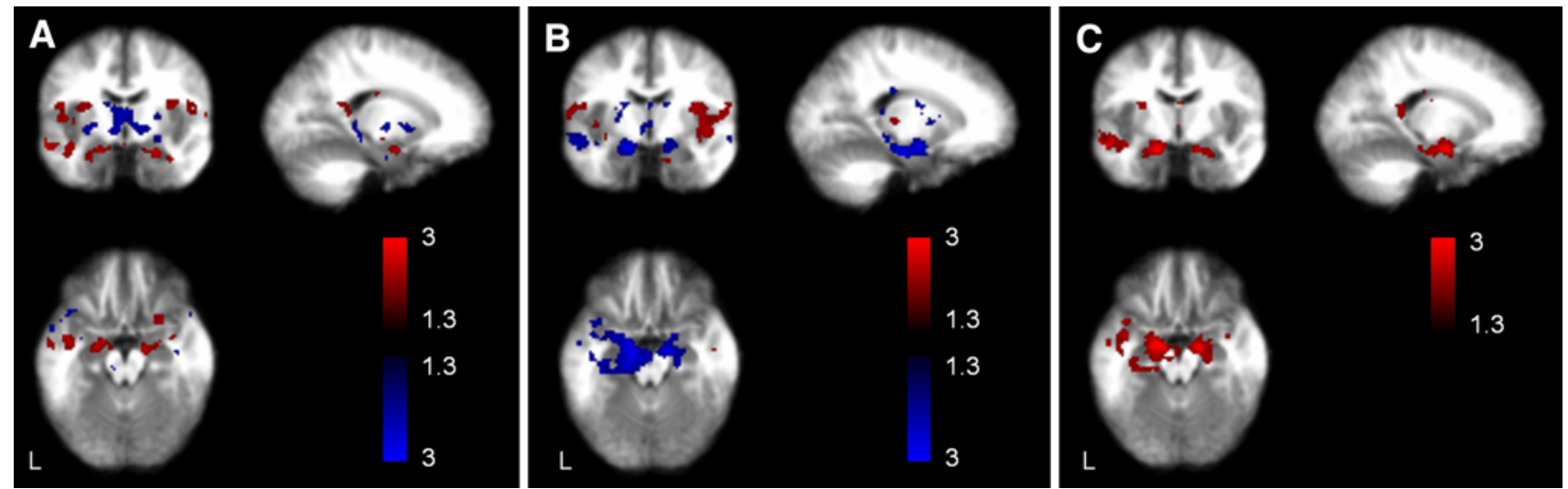

Fig. 2 a Group activation map ( $z$ scores, threshold 1.3 or $p<0.05$ ) for the short duration fearful gaze, averted versus direct: increased BOLD signal in the amygdalae. Planes correspond to $x=-18$, $y=-5, z=-17$ in MNI space, as in Fig. 1. b Same for long

Threat and gaze are thought to affect different amygdaloid nuclei (Hoffman et al. 2007), which in this study could not be separated. In future studies, the high spatial resolution available at $7 \mathrm{~T}$ in single subject studies, could be employed to further disentangle threat, gaze and stimulus duration effects.

These data help reconcile findings from previous studies, and show that stimulus duration can considerably influence the amygdala's response to threat. We interpret these data as being the result of variations in stimulus appraisal: the short percept $(300 \mathrm{~ms})$ of a fearful face looking away rapidly orients the viewer to the presence of a potential danger (Hadjikhani et al. 2008)—however, when more time is given to process the stimulus $(\geq 1,500 \mathrm{~ms})$, duration fearful gaze, averted versus direct: decreased BOLD signal in the amygdalae. c Duration effect: significant duration effects found in both amygdalae

the ambiguity surrounding the source of threat associated with direct gaze fear becomes prominent and alerting (Adams et al. 2003).

\section{Conclusion}

These data show that, provided an appropriate slice orientation is used in combination with a thin slice thickness, fMRI at ultra-high field can be used to detect BOLD signal from the amygdalae. Such acquisitions allowed a comparison of BOLD responses to averted and direct gaze fearful faces with a relatively short or long duration in a single session, confirming that the inversion of the detected 
BOLD responses is due only to the duration of the presented stimulus.

Acknowledgments This work was supported by grant FNS PP00P3_130191 to $\mathrm{NH}$, by the Centre d'Imagerie BioMédicale (CIBM) of the University of Lausanne (UNIL), the Swiss Federal Institute of Technology Lausanne (EPFL), the University of Geneva (UniGe), the Centre Hospitalier Universitaire Vaudois (CHUV), the Hôpitaux Universitaires de Genève (HUG) and the Leenaards and the Jeantet Foundations.

Open Access This article is distributed under the terms of the Creative Commons Attribution Noncommercial License which permits any noncommercial use, distribution, and reproduction in any medium, provided the original author(s) and source are credited.

\section{References}

Adams RB Jr, Gordon HL, Baird AA, Ambady N, Kleck RE (2003) Effects of gaze on amygdala sensitivity to anger and fear faces. Science 300(5625): 1536

Adams RB, Jr, Franklin RG, Jr., Kveraga K, Ambady N, Kleck RE, Whalen PJ, Hadjikhani N, Nelson AJ (2011) Amygdala responses to averted vs direct gaze fear vary as a function of presentation speed. Soc Cogn Affect Neurosci (in press)

Cristinzio C, N'Diaye K, Seeck M, Vuilleumier P, Sander D (2010) Integration of gaze direction and facial expression in patients with unilateral amygdala damage. Brain 133(Pt 1):248-261

Hadjikhani N, Hoge R, Snyder J, de Gelder B (2008) Pointing with the eyes: the role of gaze in communicating danger. Brain Cogn $68(1): 1-8$
Hoffman KL, Gothard KM, Schmid MC, Logothetis NK (2007) Facial-expression and gaze-selective responses in the monkey amygdala. Curr Biol 17(9):766-772

Marques JP, Kober T, Krueger G, van der Zwaag W, Van de Moortele PF, Gruetter R (2010) MP2RAGE, a self bias-field corrected sequence for improved segmentation and T1-mapping at high field. NeuroImage 49(2):1271-1281

Merboldt KD, Fransson P, Bruhn H, Frahm J (2001) Functional MRI of the human amygdala? NeuroImage 14(2):253-257

N'Diaye K, Sander D, Vuilleumier P (2009) Self-relevance processing in the human amygdala: gaze direction, facial expression, and emotion intensity. Emotion 9(6):798-806

Norbury R, Selvaraj S, Taylor MJ, Harmer C, Cowen PJ (2010) Increased neural response to fear in patients recovered from depression: a $3 \mathrm{~T}$ functional magnetic resonance imaging study. Psychol Med 40(3):425-432

Ohrmann P, Rauch AV, Bauer J, Kugel H, Arolt V, Heindel W, Suslow T (2007) Threat sensitivity as assessed by automatic amygdala response to fearful faces predicts speed of visual search for facial expression. Exp brain Res 183(1):51-59

Robinson S, Windischberger C, Rauscher A, Moser E (2004) Optimized $3 \mathrm{~T}$ EPI of the amygdalae. NeuroImage 22(1): 203-210

Speck O, Stadler J, Zaitsev M (2008) High resolution single-shot EPI at $7 \mathrm{~T}$. Magma 21(1-2):73-86

van der Zwaag W, Francis S, Head K, Peters A, Gowland P, Morris P, Bowtell R (2009) fMRI at 1.5, 3 and 7 T: characterising BOLD signal changes. NeuroImage 47(4):1425-1434

Yacoub E, Shmuel A, Pfeuffer J, Van De Moortele PF, Adriany G, Andersen P, Vaughan JT, Merkle H, Ugurbil K, Hu X (2001) Imaging brain function in humans at 7 Tesla. Magn Reson Med 45(4):588-594 